\title{
Measuring X-ray anisotropy in solar flares. Prospective stereoscopic capabilities of STIX and MiSoIFA
}

\author{
Diego Casadei ${ }^{1}$, Natasha L. S. Jeffrey ${ }^{2}$, and Eduard P. Kontar ${ }^{2}$ \\ 1 University of Applied Sciences \& Arts Northwestern Switzerland (FHNW), Bahnhofstrasse 6, 5210 Windisch, Switzerland \\ e-mail: diego.casadei@fhnw.ch \\ 2 School of Physics \& Astronomy, University of Glasgow, Glasgow G12 8QQ, UK
}

Received 15 February 2017 / Accepted 28 July 2017

\begin{abstract}
Context. During a solar flare, a large percentage of the magnetic energy released goes into the kinetic energy of non-thermal particles, with X-ray observations providing a direct connection to $\mathrm{keV}$ flare-accelerated electrons. However, the electron angular distribution, a prime diagnostic tool of the acceleration mechanism and transport, is poorly known.

Aims. During the next solar maximum, two upcoming space-borne X-ray missions, STIX on board Solar Orbiter and MiSolFA, will perform stereoscopic X-ray observations of solar flares at two different locations: STIX at 0.28 AU (at perihelion) and up to inclinations of $\sim 25^{\circ}$, and MiSolFA in a low-Earth orbit. The combined observations from these cross-calibrated detectors will allow us to infer the electron anisotropy of individual flares confidently for the first time.

Methods. We simulated both instrumental and physical effects for STIX and MiSolFA including thermal shielding, background and $\mathrm{X}$-ray Compton backscattering (albedo effect) in the solar photosphere. We predict the expected number of observable flares available for stereoscopic measurements during the next solar maximum. We also discuss the range of useful spacecraft observation angles for the challenging case of close-to-isotropic flare anisotropy.

Results. The simulated results show that STIX and MiSolFA will be capable of detecting low levels of flare anisotropy, for M1-class or stronger flares, even with a relatively small spacecraft angular separation of $20-30^{\circ}$. Both instruments will directly measure the flare X-ray anisotropy of about $40 \mathrm{M}$ - and X-class solar flares during the next solar maximum.

Conclusions. Near-future stereoscopic observations with Solar Orbiter/STIX and MiSolFA will help distinguishing between competing flare-acceleration mechanisms, and provide essential constraints regarding collisional and non-collisional transport processes occurring in the flaring atmosphere for individual solar flares.
\end{abstract}

Key words. Sun: flares - Sun: X-rays - Sun: atmosphere - space vehicles: instruments

\section{Introduction}

Solar flares are the most powerful explosive events in the solar system, and large flares can release up to $10^{32} \mathrm{erg}$ of energy in a few minutes (Benz 2008; Holman et al. 2011). A large fraction of this energy, stored in coronal magnetic fields and released by magnetic reconnection, goes into the acceleration of particles. However, the mechanisms transforming magnetic energy into kinetic energy are still not clearly understood. Flare-accelerated electrons emit a continuous spectrum of bremsstrahlung X-rays that can span a wide energy range up to gamma rays. Hard $\mathrm{X}$-rays (HXR, $\gtrsim 20 \mathrm{keV}$ ) are a direct link to flare-accelerated electrons and a vital probe of the flare physical processes occurring at the Sun (e.g. Brown et al. 2003; Kontar et al. 2011; Holman et al. 2011).

Current X-ray observations are performed by the spaceborne Reuven Ramaty High Energy Solar Spectroscopic Imager (RHESSI; Lin et al. 2002), using nine rotation modulation collimators and bulk germanium (Ge) detectors to perform indirect imaging and spectroscopy from $3 \mathrm{keV}$ to $17 \mathrm{MeV}$ with an angular resolution of few arcsec (Hurford et al. 2002). The brightest X-ray sites are often found at the footpoints of newly reconnected magnetic loops that link coronal acceleration regions with the much denser chromosphere. Here, the bulk of the accelerated electrons interact, losing energy via electron-electron
Coulomb collisions and emitting bremsstrahlung X-rays by interacting with the ambient ions. Solar flare X-ray observations typically show a superposition of two distributions. The first is a thermal component emanating from the corona, with flare temperatures of a few tens of million degrees, dominating up to $10-20 \mathrm{keV}$. The second is a non-thermal power law extending to higher energies with a spectral index in the range from $\sim 2$ to 5 for HXR footpoints sources and, when detected, from $\sim 3$ to 8 for the coronal non-thermal emission (Emslie et al. 2003; Kašparová et al. 2005; Krucker \& Lin 2008; Simões \& Kontar 2013; Chen \& Petrosian 2013).

Although the flare X-ray energy spectrum is well observed by RHESSI, the angular distribution is poorly constrained. The X-ray spectrum is dependent on the angular distribution of the parent electrons (e.g. Massone et al. 2004; Kontar et al. 2011) and uncertainty regarding the electron pitch-angle distribution can also lead to changes in inferred plasma parameters, e.g. an overestimation of coronal density from X-ray imaging (Jeffrey et al. 2014). Thus, knowing the directivity of both the injected and radiating electron distributions is essential for understanding the type of acceleration mechanism(s) and the transport and interactions of solar flare electrons. Often, in an oversimplified collisional thick-target model, the injected and emitting electrons are assumed to be beamed along the guiding field lines (e.g. Brown 1971). However acceleration 
(for example by a second-order Fermi process) might produce an isotropic distribution of accelerated electrons (e.g. Melrose 1994; Miller et al. 1996; Petrosian 2012). Furthermore, electron transport through the surrounding solar plasma ultimately broadens the electron distribution, increasing the isotropy by collisional or non-collisional pitch-angle scattering (Kontar et al. 2014). Therefore, even if the injected distribution is strongly beamed, the angular distribution of radiating electrons is expected to isotropise as they are transported from the corona to the chromosphere.

So far, HXR directivity has been studied with the following techniques (e.g. Kontar et al. 2011, as a review):

1. Statistical flare studies of centre-to-limb variations in flux or spectral index (e.g. Ohki 1969; Kašparová et al. 2007).

2. Albedo mirror analysis of strong solar flares (Kontar \& Brown 2006; Dickson \& Kontar 2013).

3. Linear X-ray polarization measurements from a single flare with one satellite (e.g. Tindo et al. 1970; McConnell et al. 2004; Suarez-Garcia et al. 2006).

4. Simultaneous observations of a single flare with two satellites at different viewing angles. (e.g. Kane 1981; Hurley 1986; Kane et al. 1988, 1998; McTiernan \& Petrosian 1990).

Method 2 suggests that the HXR emitting electron distribution is close to isotropic, and not beamed as in a simple standard flare model, at least for the few events published. This method uses the X-ray albedo effect (e.g. Tomblin 1972; Santangelo et al. 1973; Bai \& Ramaty 1978), where sunwards emitted X-rays are Compton backscattered in the photosphere into the observer direction. The directivity is then determined by separating the directly emitted and reflected components of HXR flux that contribute to the observed X-ray spectrum. Kontar \& Brown (2006) studied two flares and their analysis showed that both flares were close to isotropic. A follow-up study of eight events by Dickson \& Kontar (2013) again found a lack of electron anisotropy below $150 \mathrm{keV}$.

Recently Kašparová et al. (2007) studied 398 flares using method 1, accounting for the albedo component. Although they found changes in spectral index that were consistent with the presence of an albedo component, the statistical study gave no clear conclusion regarding average flare directivity.

Method 3 uses the direct link between X-ray linear polarization and electron anisotropy. Electron directivity and Xray polarization have been extensively modelled (with and without albedo) for different scenarios (e.g. Leach \& Petrosian 1983; Bai \& Ramaty 1978; Emslie et al. 2008; Jeffrey \& Kontar 2011). Nevertheless, observations with past instruments and non-dedicated polarimeters, such as RHESSI, have proved inconclusive, owing to instrumental issues (small effective area etc.) inducing large uncertainties associated with the measurements. The recently launched POLAR (Hajdas 2015), a wide field-of-view X-ray polarimeter installed on the Chinese space station Tiangong-2 in September 2016, should allow for confident detection of X-ray polarization during large flares at suitable heliocentric angles away from the solar disk centre. Importantly, POLAR has an effective area of $200 \mathrm{~cm}^{2}$, i.e. two orders of magnitude larger than the polarimeter on board RHESSI, and a low minimum detectable polarization of $5 \%$, that should remove some of the previous issues (e.g. high background levels and large uncertainties). However, POLAR will be operational during a period of decreasing solar activity.

Unambiguous measurements of solar flare electron anisotropy can be obtained through X-ray directivity measurements made by cross-calibrated detectors looking at the same source from two separate points of view (method 4). Such previous stereoscopic studies (e.g. Kane et al. 1998) found no clear evidence for directivity at large X-ray energies. However, past direct measurements by multiple spacecrafts suffered greatly from calibration issues, owing to the use of different types of detectors, therefore the results were questionable at best. Thus, it is fundamental that the two instruments have a well-known energy cross-calibration.

A concrete possibility of obtaining simultaneous stereoscopic observations will be provided by two instruments, which will operate at the next solar maximum. The Spectrometer Telescope for Imaging X-rays (STIX; Krucker et al. 2013), is an instrument to be flown on board the ESA/NASA Solar Orbiter mission (Müller et al. 2013) within the ESA Cosmic Vision programme. Solar Orbiter will be launched in October 2018 and it will start its science programme after a three year cruise phase. The Micro Solar-Flare Apparatus (MiSolFA; Casadei 2014) is a compact X-ray detector being developed in Switzerland, in collaboration with the French STIX team and the Italian Space Agency, to be operated in low-Earth polar orbit at the next solar maximum. Thus, during the next solar maximum period simultaneous observations will be jointly performed by STIX and MiSolFA, the first orbiting around the Sun and the second around the Earth. Importantly, both instruments will adopt the same type of photon detectors, overcoming the calibration issues of past instruments.

The purpose of this paper is to discuss the prospective stereoscopic observations with STIX and MiSolFA, highlighting the capabilities of both instruments working in tandem, and estimating the number of observable flares, which are suitable for measuring the anisotropy of the X-ray emission. The two instruments are illustrated in Sect. 2. Simulated solar flares with small differences in electron anisotropy are studied, and we consider both instrumental and physical effects such as X-ray albedo. In Sect. 3 it is shown that, even with a spacecraft angular separation as small as $20-30^{\circ}$, a mildly anisotropic distribution will produce detectable differences in the observed X-ray spectra, for a bright enough solar flare. The expected number of flare observations is finally estimated in Sect. 4, where it is found that several flares per year will be suitable for an energy-dependent directivity measurement with STIX and MiSolFA. In Sect. 5, all the main results are summarized.

\section{Dual observations with STIX and MiSoIFA}

The STIX instrument will provide X-ray imaging spectroscopy from 4 to $150 \mathrm{keV}$ with 32 Caliste-SO units equipped with CdTe crystals (Meuris et al. 2012) and energy resolution better than $1 \mathrm{keV}$ (FWHM) from 14 to $60 \mathrm{keV}$. The imaging is performed with an indirect technique based on the Moiré effect, achieving an angular resolution of about 7 arcsec. At perihelion, STIX will observe flares from a distance three times closer to the Sun than instruments orbiting around the Earth, hence achieving an effective spatial resolution similar to RHESSI (which has $\sim 2$ arcsec resolution). In order to complement STIX observations with minimal differences in the energy response, MiSolFA will adopt the same photon detectors, i.e. Caliste units equipped with CdTe crystals with $1 \mathrm{~mm}$ thickness. The orbital inclination of STIX will increase over time up to $25^{\circ}$ or more (depending on the mission duration). Hence, the two instruments will be able to observe the same flare stereoscopically from two different points of view.

The STIX and MiSolFA instruments both need to exploit indirect imaging techniques because they cannot accommodate 
grazing-incidence focussing optics, which require large focal distances of several metres. For example, astronomical direct $\mathrm{X}$-ray imagers such as the Nuclear Spectroscopic Telescope Array $(N U S T A R)$ have a $10 \mathrm{~m}$ focal length (Harrison et al. 2013). Like RHESSI, STIX and MiSolFA rely on the Moiré effect, which is produced by a pair of parallel grids placed in front of each photon detector. The STIX instrument has 30 pairs of tungsten grids providing Fourier components with 9 different directions (at $20^{\circ}$ steps) and 10 angular scales (from 7 to 950 arcsec, with constant-ratio steps of $\sqrt{2}$ ). Hence, STIX will be able to precisely locate the flare on the Sun and study the morphology of the X-ray emitting sources. On the other hand, the main purpose for the imaging system of MiSolFA is to separate the flare HXR footpoints, relying on other observations to locate the source on the Sun. For this purpose, it is sufficient to sample a relatively narrow angular range (from 10 to 60 arcsec). MiSolFA will cover this range with 12 subcollimators sampling two orthogonal directions with frequencies following a Fourier series. Therefore, although only in a very limited angular range, MiSolFA will have a better point spread function than STIX, whereas the latter will be able to cover a much wider angular range and will be able to locate the source with very high precision (of order of 1 arcsec).

In the rest of this paper, we focus on X-ray spectroscopy alone, hence the imaging performance of the two instruments is ignored, apart from taking into account the reduction of effective area (because of the grids, only about $25 \%$ of the photons reach the detectors). The effective area of MiSolFA detectors is $40 \%$ of the STIX area. In addition, the time average over the higheccentricity orbit of STIX gives it almost a factor 3 increase in intensity, owing to the closer distance from the Sun. This gives a ratio of 7.5 between STIX and MiSolFA acceptances. We also account for additional details in the following simulations. Arriving photons "see" the thermal shield of each instrument, which is designed to stop radiation up to the X-ray range. The STIX instrument has two beryllium (Be) windows with total thickness of $3 \mathrm{~mm}$, whereas a $0.3 \mathrm{~mm}$ thick Be layer has been considered for MiSolFA.

In order to have sufficient counts up to about hundred $\mathrm{keV}$, flares of class $\mathrm{M}$ or above are considered. This allows for the precise determination of the thermal and non-thermal contributions to the total flux. To avoid large dead times owing to the high flux of low-energy photons, STIX employs a movable aluminium attenuator with $0.6 \mathrm{~mm}$ thickness. On the other hand MiSolFA has no movable part, as this would compromise the pointing stability of such a light satellite. Nevertheless, the low-energy flux is also attenuated by MiSolFA, which plans to adopt golden grids fabricated onto a silicon or carbon substrate. Here a Si layer is considered (a conservative assumption), which absorbs most photons below $8 \mathrm{keV}$. Hence the MiSolFA transmission is not very different from what STIX achieves with the attenuator in front of the photon detectors (see Fig. 1).

Furthermore, although STIX and MiSolFA will adopt the same photon detectors, their performance cannot be expected to be identical, since Solar Orbiter will only start collecting science data after a cruise of three years. During this initial phase, the CdTe crystals will experience some ageing owing to incident radiation. The radioactive sources installed on these detectors will provide continuous calibration data, which is fundamental to ensure a good cross-calibration. Here it is conservatively estimated that the STIX resolution will worsen by a factor of two (a larger effect than what found by Eisen et al. 2002; Zanarini et al. 2004), whilst no ageing is considered for MiSolFA.

Finally, the background counts will have different distributions for the two detectors. One example is shown in Fig. 2,

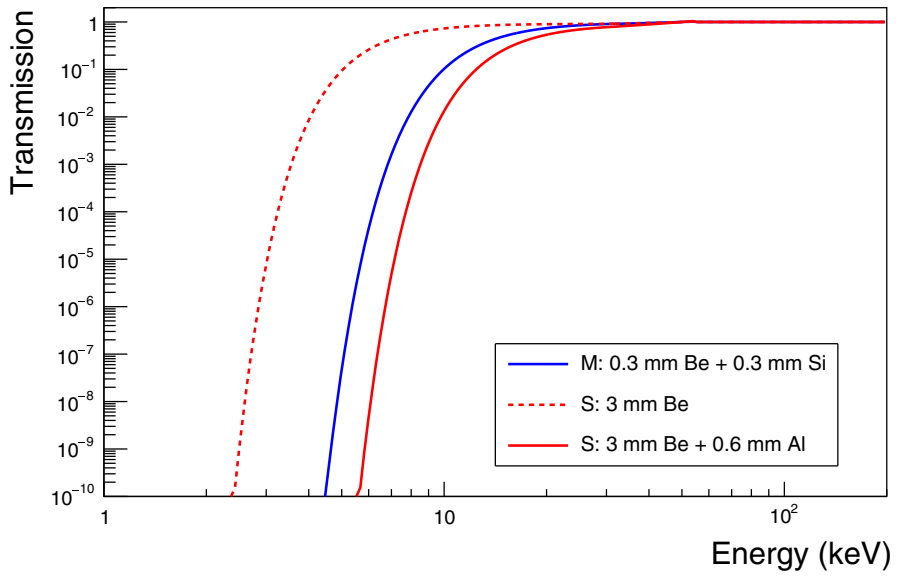

Fig. 1. Transmission of the passive material in front of STIX (S) and MiSolFA (M) X-ray detectors. STIX employs a movable Al attenuator with $0.6 \mathrm{~mm}$ thickness and two Be windows with total thickness of $3 \mathrm{~mm}$, while MiSolFA has a $0.3 \mathrm{~mm}$ Si layer and a Be $0.3 \mathrm{~mm}$ layer.

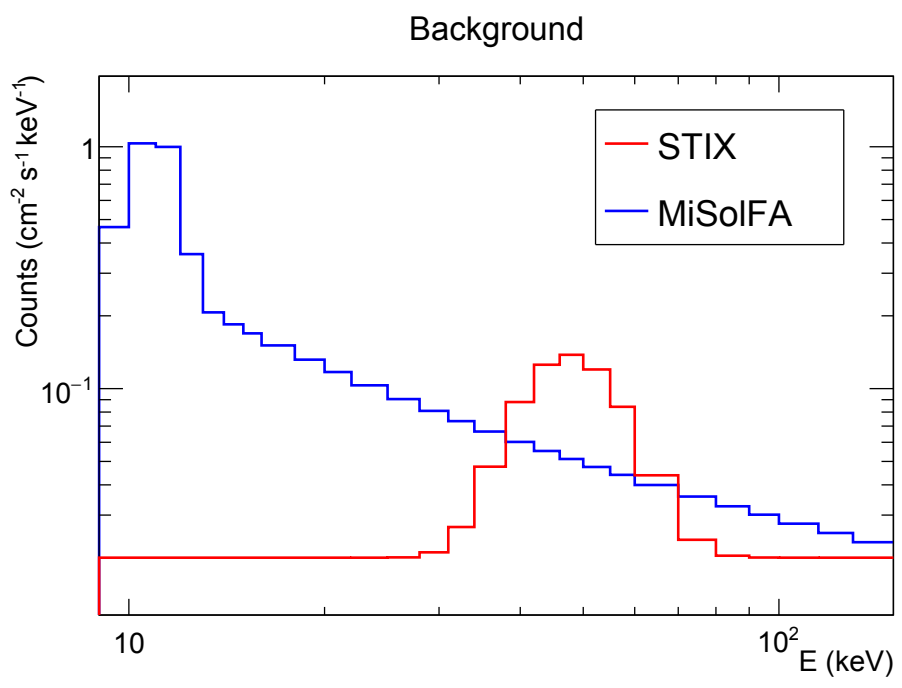

Fig. 2. Estimated background counts for STIX and MiSolFA. The actual background counts for STIX and MiSolFA will be measured once both instruments are operating.

where the MiSolFA background distribution is taken to be similar to the RHESSI background at the beginning of the mission, and for STIX a flat component is superimposed with a bump that mimics the background X-ray radiation (Marshall et al. 1980). The actual background distributions will be measured once the two instruments start operating. Here what matters is that they are expected to have different shapes, affecting the measured photon spectra in different ways.

Flare spectra are rapidly falling energy distributions, while the background counts have a more uniform distribution. Therefore, at high energies (e.g. well above $100 \mathrm{keV}$ ) the measurement will be background dominated. On the other hand, the low-energy part is dominated by the thermal emission, which is not expected to show significant anisotropy, although a thermal source can show low levels (few percent) of directivity and polarization (e.g. see Emslie \& Brown 1980). Hence the useful energy range for directivity studies extends from the end of the thermal region $(\sim 20 \mathrm{keV})$, up to the energy bins in which the background counts are of the same order of magnitude as the actual flare 


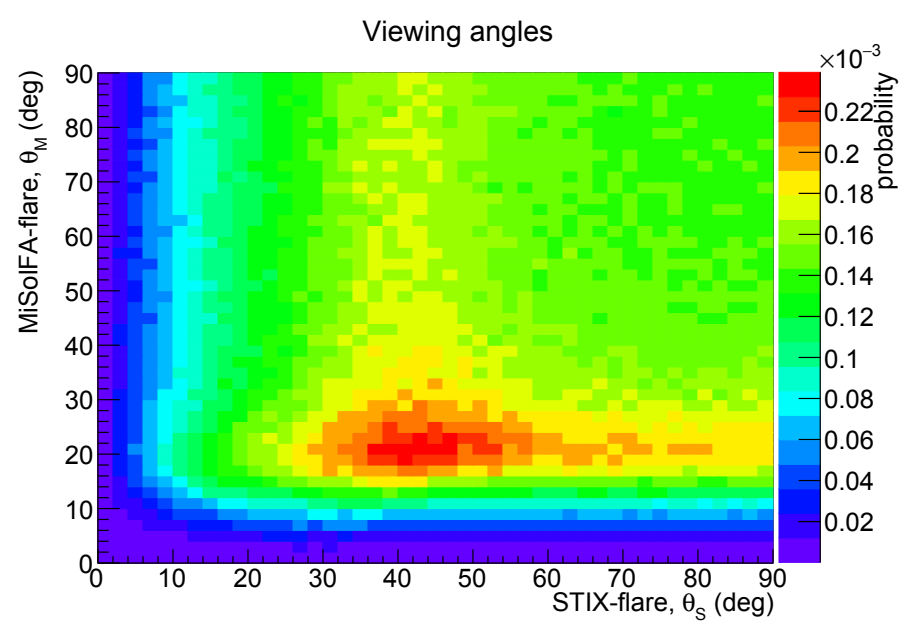

Fig. 3. Viewing angles of STIX $\left(\theta_{\mathrm{S}}\right)$ and $\operatorname{MiSolFA}\left(\theta_{\mathrm{M}}\right)$. The most probable ranges of viewing angles are $\theta_{\mathrm{S}} \sim 30-50^{\circ}$ and $\theta_{\mathrm{M}} \sim 20-30^{\circ}$.

photon rate. For example, for M-class flares this energy region roughly goes from 20 to $100 \mathrm{keV}$.

In order to estimate the flare viewing angles of STIX and MiSolFA, the flare position on the Sun was simulated according to the measured distribution seen by RHESSI over the past decade $^{1}$. Similar to the sunspot distribution, flares are uniform in solar longitude, which implies a higher density of flares approaching the limb when looking from the Earth, and bimodal in latitude, with peaks at about $\pm 13.5^{\circ}$ and a root mean square of $6.3^{\circ}$. With MiSolFA in a low Earth orbit and STIX taken approximately ${ }^{2}$ uniform in latitude (within $\pm 0.5 \mathrm{rad}$ ) and in longitude (within $\pm \pi \mathrm{rad}$ ), there is about $50 \%$ probability (ignoring beyond-the-limb flares ${ }^{3}$ ) that a flare is visible by one instrument, hence the integral of the distribution in Fig. 3 is 0.25 , which is the fraction of flares visible by both instruments. There is a low probability for small viewing angles with a peak at $\theta_{\mathrm{S}} \sim 30-50^{\circ}$ for STIX and $\theta_{\mathrm{M}} \sim 20-30^{\circ}$ for MiSolFA; this is about $40 \%$ higher than a broad plateau reaching $90^{\circ}$, which corresponds to limb flares. Accounting for a $20 \%$ live time for STIX, corresponding to the fraction of time it will spend in science mode during the main phase of Solar Orbiter ${ }^{4}$ and for a fraction of flares visible by both instruments equal to $50 \%$, one expects $\mathrm{Mi}$ SolFA to observe the same event as STIX for $10 \%$ of all flares. The MiSolFA instrument has a two year nominal science mission duration, and this amounts to 73 days of net observing time. Assuming 20\% dead time, caused by some issue for at least one of the instruments, one ends up with two months of total simultaneous observing time.

\section{Simulation of flare measurement}

Starting from different electron distributions, Jeffrey \& Kontar (2011) computed the X-ray bremsstrahlung emission as a function of the electron energy and estimated the total X-ray flux along all directions, for different degrees of electron anisotropy.

\footnotetext{
1 http://hesperia.gsfc.nasa.gov/hessidata/dbase/hessi_ flare_list.txt

2 The orbit eccentricity is neglected here.

$310-11 \%$ of all flares observed by each instrument fall beyond the limb by no more than $10^{\circ}$, which leaves their coronal source visible. Beyond this angle a flare is fully occulted.

4 This conservatively assumes no attempt to optimize the overlapping time with MiSolFA.
}

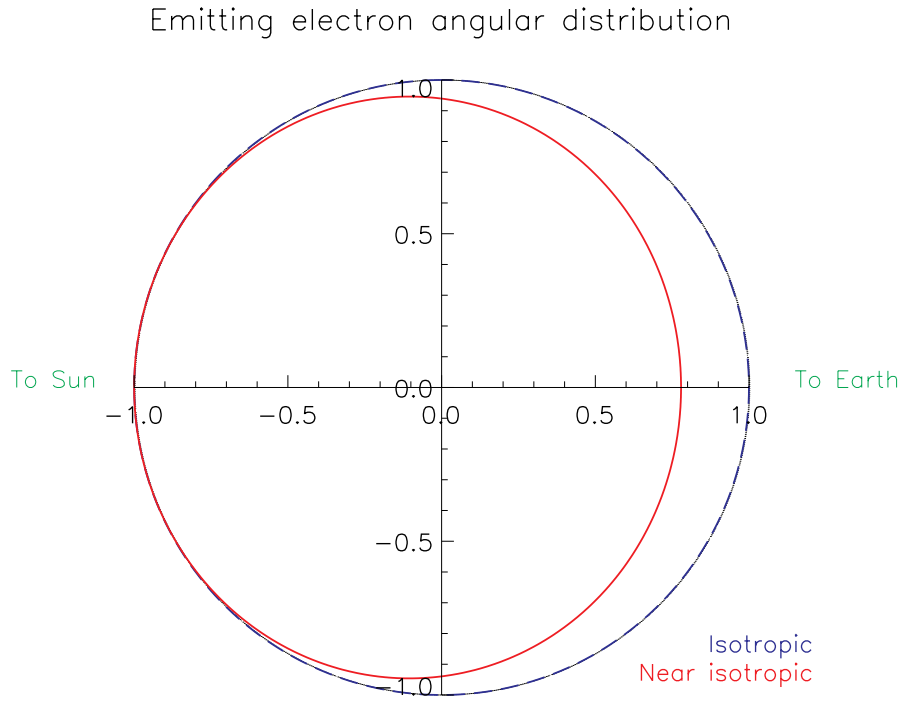

Fig. 4. Polar plot of the simulated electron angular distributions for completely isotropic (blue) and mildly anisotropic (red). In the simple model considered here, the energy dependence of the electron anisotropy is neglected.

Importantly, this work also included the X-ray albedo component, where photons emitted towards the Sun are Compton backscattered in the photosphere towards the observer with changed photon properties. The albedo component produces a bump in the energy spectrum between $\sim 10-100 \mathrm{keV}$ and this bump must be included since it changes all the measured electron and photon properties, including anisotropy. In our simulation, we only modelled the non-thermal electron distribution. The resulting X-ray bremsstrahlung (including the albedo component) is calculated for a HXR footpoint source located at a chromospheric height of $1 \mathrm{Mm}$ above the photosphere (for more information see Kontar \& Jeffrey 2010; Jeffrey \& Kontar 2011). The energy spectrum of the emitting electrons is a single power law with spectral index of 2 , hence the injected electron distribution has a spectral index of $\delta \sim 4$ and the emitting photon distribution has a spectral index of $\gamma \sim 3$. Here we make a comparison between an isotropic and a mildly anisotropic case with a Gaussian pitch-angle distribution with approximately $0.4 \mathrm{rad}$ standard deviation.

In the simulation, we use a simple model where the angular distribution of the electrons does not change with energy, since more complicated electron distributions are not required to show the prospective stereoscopic capabilities of both instruments. Here we focus on the physically relevant observable differences in the detected X-ray flux, which arise from different lines of sight, albedo, and instrumental effects. The latter are independent of the details of the parent electron distribution, while both direct and reflected emissions are expected to be functions of the electron energy. However, while the energy dependence of the albedo component is well understood (Jeffrey \& Kontar 2011), the "true" electron distribution is not known and likely changes in different flares. Hence there was no attempt to model the full complexity of real flares, as this is not necessary to assess the capability of the two instruments to perform joint measurements from which the anisotropy can be inferred, whatever the underlining electron model is. The angular distributions of the X-ray emitting electrons used in our analysis are shown in Fig. 4. The non-isotropic distribution is only slightly sunwards beamed compared to the isotropic case. 
Ratio of photon flux: small anisotropy/isotropic

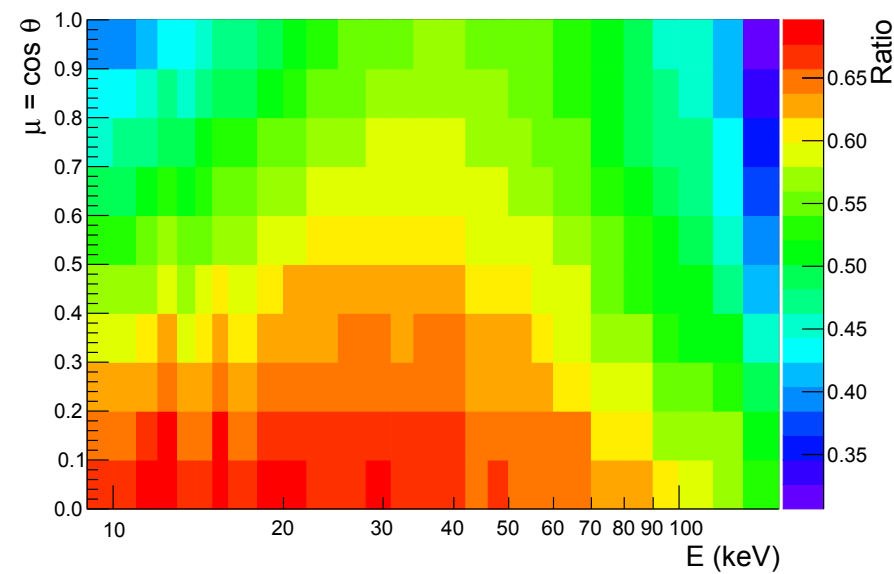

Fig. 5. Binwise ratio between photons emitted by mildly anisotropic and fully isotropic electron distributions. The isotropic thermal component is neglected in this plot.

The two corresponding photon spectra have different energy dependence along different directions. Figure 5 shows their binwise ratio as a function of the energy and $\mu \equiv \cos \theta$, where $\theta=\theta_{\mathrm{S}}$ or $\theta_{\mathrm{M}}$ is the viewing angle with respect to the local flare vertical direction (equal to the local heliocentric angle on the solar disk). This means that, unless STIX and MiSolFA are symmetrically located with respect to the flare direction (which is very unlikely, see Fig. 3), they will measure different energy spectra. Figure 6 shows the difference $\left|\cos \theta_{\mathrm{S}}-\cos \theta_{\mathrm{M}}\right|$ between STIX and MiSolFA: most flares will be observed with angular separations large enough to measure sizeable differences in the photon spectra. Here we take $\cos \theta_{\mathrm{S}}=0.7-0.8$ for STIX and $\cos \theta_{\mathrm{M}}=$ 0.9-1.0 for MiSolFA, to make a concrete example. We used this conservative example since a larger difference in the viewing angles will generally produce a greater difference in the resulting energy spectra observed by both instruments.

The most effective way of detecting deviations from a fully isotropic electron distribution is to look for differences in the shape (not only in the normalization) of the energy spectrum of the emitted X-rays. A sizable difference in the absolute flux reaching the two detectors is expected. However, it is very difficult to establish a standard candle for calibration of absolute fluxes. On the other hand, shape differences can be compared against spectra, which can be safely assumed to have the same shape, for example when the same source is viewed at about the same angle. This is why checking for shape differences is a more robust approach from the experimental point of view. The example under consideration, i.e. minimal variations in anisotropy, represents a challenging case, because it implies choosing adjacent slices in Fig. 5, with minimal shape differences in the energy spectrum. Figure 7 shows the corresponding "true" energy spectra of the simulated photon flux towards STIX and MiSolFA, using the same energy binning for both.

After having considered the passage through all materials in front of the photon detectors, the different energy resolution and background distributions, one obtains the expected distributions shown in Fig. 8. Next, they are taken as the input for a pseudo-experiment, in which the expected (real) value in each bin is taken as the parameter of a Poisson distribution, which is adopted to generate a random (integer) number of counts in 1 min of observation time for both detectors. The result is shown in Fig. 9.
STIX - MiSolFA

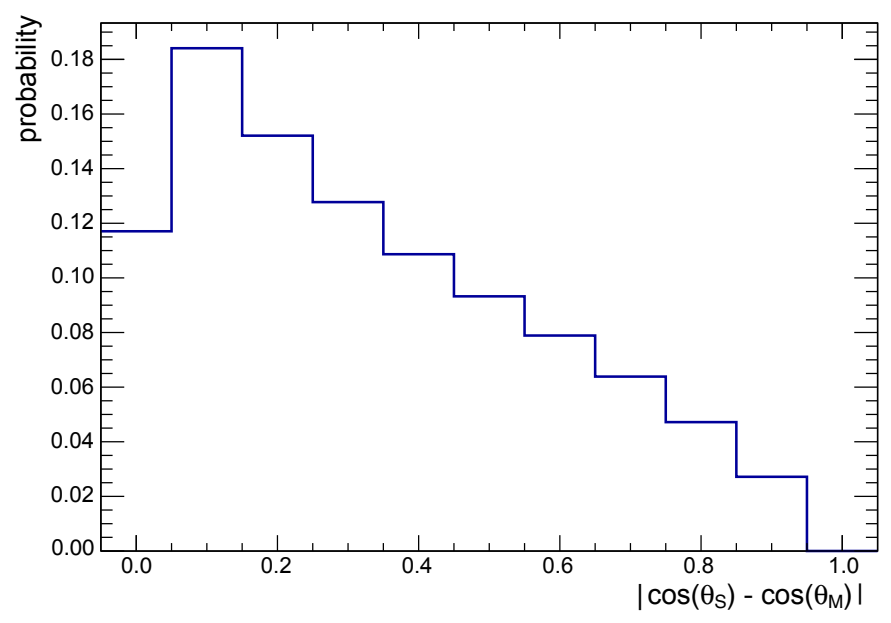

Fig. 6. Difference in viewing angles.

Figures 7 to 9 show two distributions for each instrument, one corresponding to the fully isotropic electron distribution and one obtained with the mild anisotropy shown in Fig. 4. Error bars reflect the finite size of the Monte Carlo sample in Figs. 7 and 8, while Fig. 9 also includes the uncertainty from the finite size of the detected sample. The main goal is to assess how well these two cases can be distinguished, by comparing the observations performed with two instruments. In order to quickly verify that the two models can indeed be disentangled, we take here the binwise ratios between the measured distributions by MiSolFA and STIX and compare these ratios in Fig. 10. These ratios are clearly different, which implies that the two models can be distinguished by the simultaneous measurement performed with the two instruments. Hence, any greater level of flare anisotropy, if present, will also be measured by both instruments.

Actually, a better approach would be to compare the unfolded distributions, which are obtained after taking into account all detector effects and represent our best inference on the "true" input of each instrument, and then take the ratio of the unfolded distributions. However, the accuracy of the unfolding procedure is ultimately limited by the statistical uncertainty, which is shown by the error bars in Fig. 10. Hence the latter provides sufficient information to verify that the two models produce shape differences in the measured energy spectra of STIX and MiSolFA.

\section{Expected number of good flares}

Based on the statistical study of three years of RHESSI solar flare observations performed by Battaglia et al. (2005), a simulation was performed, with the purpose of understanding the range of possible scenarios to be encountered by STIX. Two functional relationships based on this study have been heuristically obtained from the simulation. The first relates the energy threshold $E_{\text {thr }}$ at which the non-thermal contribution equals the thermal component to the flare intensity $I$, taken as the logarithm in base 10 of the GOES class normalized to X1 (i.e. $I=0$ for X1, $I=-1$ for $\mathrm{M} 1, I=-2$ for $\mathrm{C} 1$, etc.). A quadratic fit with the function $E_{\mathrm{thr}}=p_{0}+p_{1} I+p_{2} I^{2}$ provides a very good description of this relationship, with best-fit parameters $p_{0}=36.3 \pm 0.07 \mathrm{keV}$, $p_{1}=3.57 \pm 0.06 \mathrm{keV}$, and $p_{2}=-0.301 \pm 0.015 \mathrm{keV}$. The fit quality is very good, with $\chi^{2}=0.072$ over 4 degrees of freedom. However one must be aware that the flare-to-flare variations are so big that the good fit quality is mostly because of the large spread among the data. 
Input on STIX, $\mu=0.7-0.8$

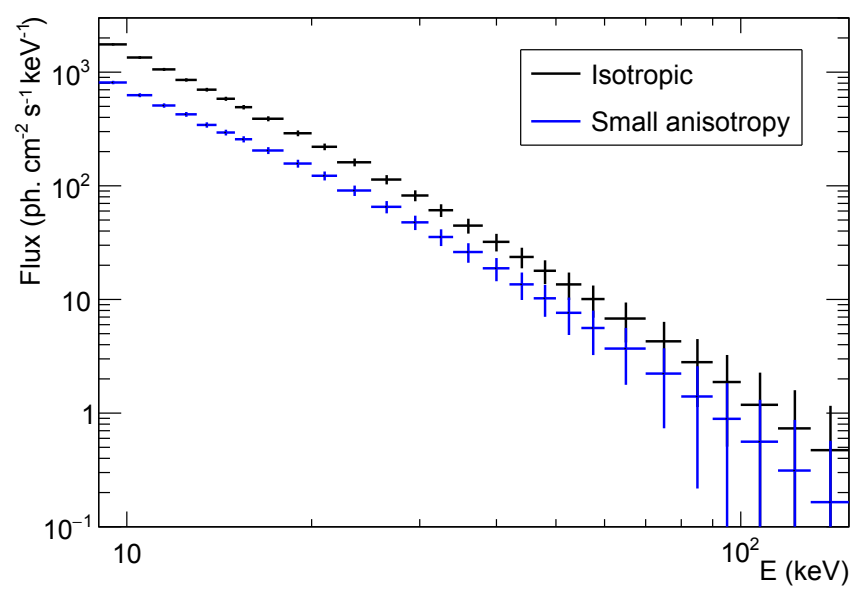

Input on MiSolFA, $\mu=0.9-1$

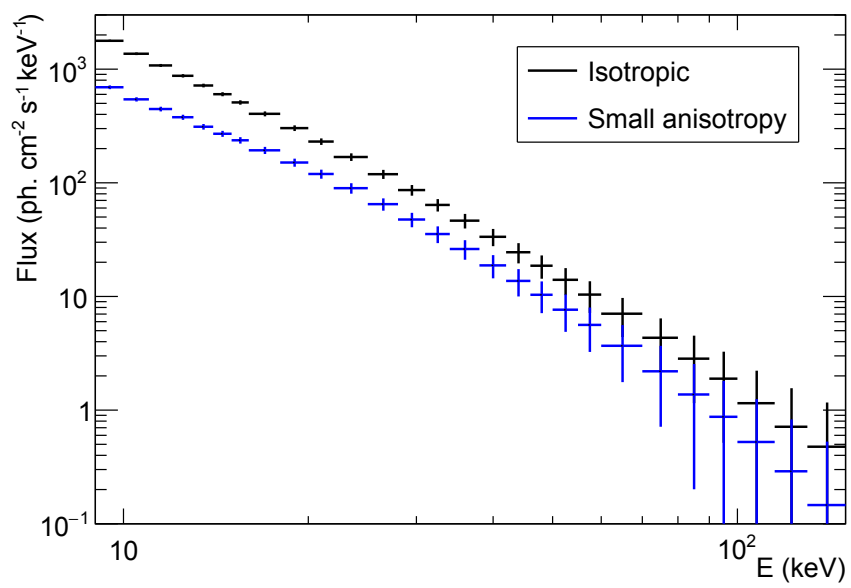

Fig. 7. Simulated input spectra with $\cos \theta_{\mathrm{S}}=0.7-0.8$ for STIX and $\cos \theta_{\mathrm{M}}=0.9-1.0$ for MiSolFA.

Expected spectrum of $\mathrm{S}$

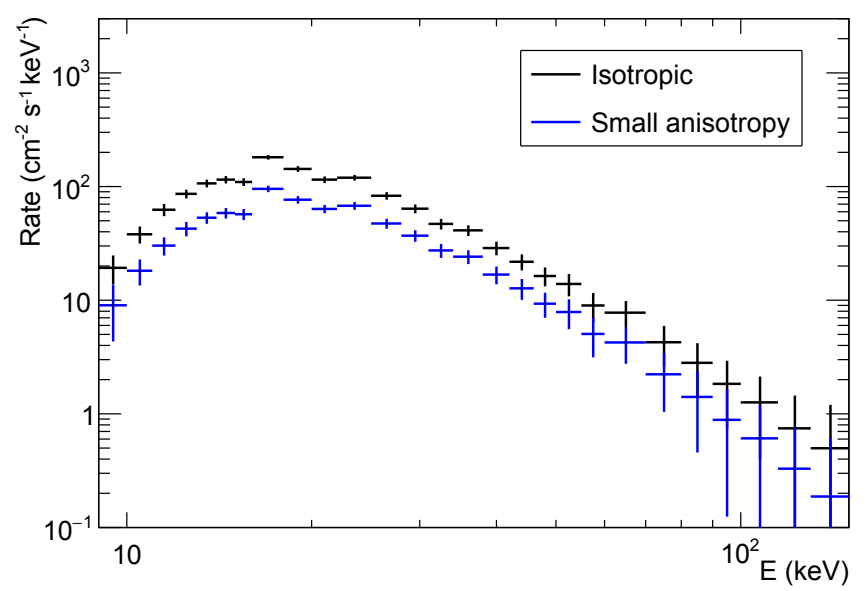

Expected spectrum of $\mathrm{M}$

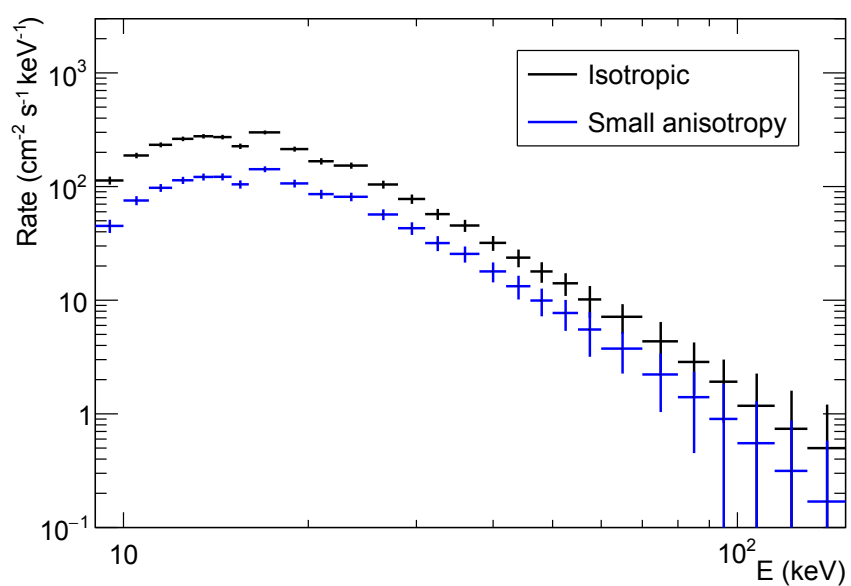

Fig. 8. Expected energy spectra with $\cos \theta_{\mathrm{S}}=0.7-0.8$ for STIX and $\cos \theta_{\mathrm{M}}=0.9-1.0$ for MiSolFA.

Photons detected by STIX in $60 \mathrm{~s}$

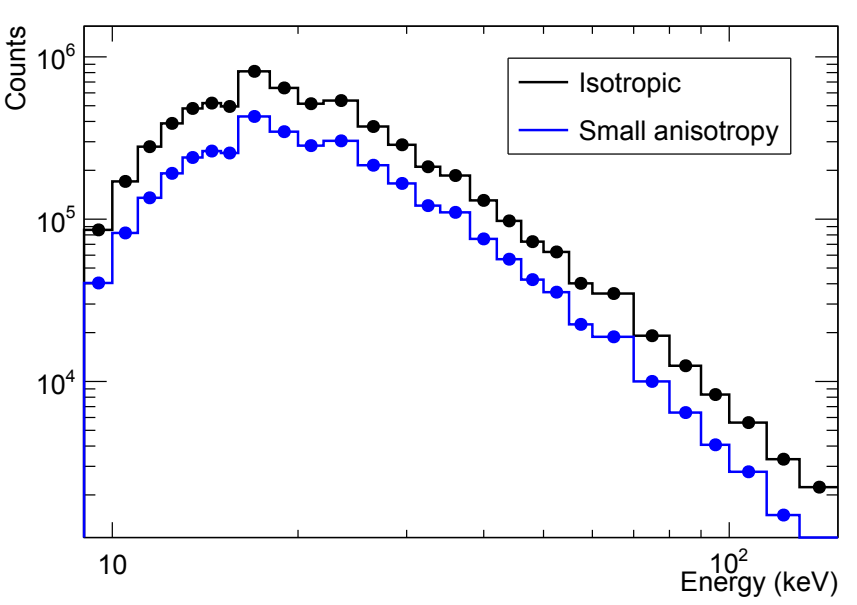

Photons detected by MiSolFA in $60 \mathrm{~s}$

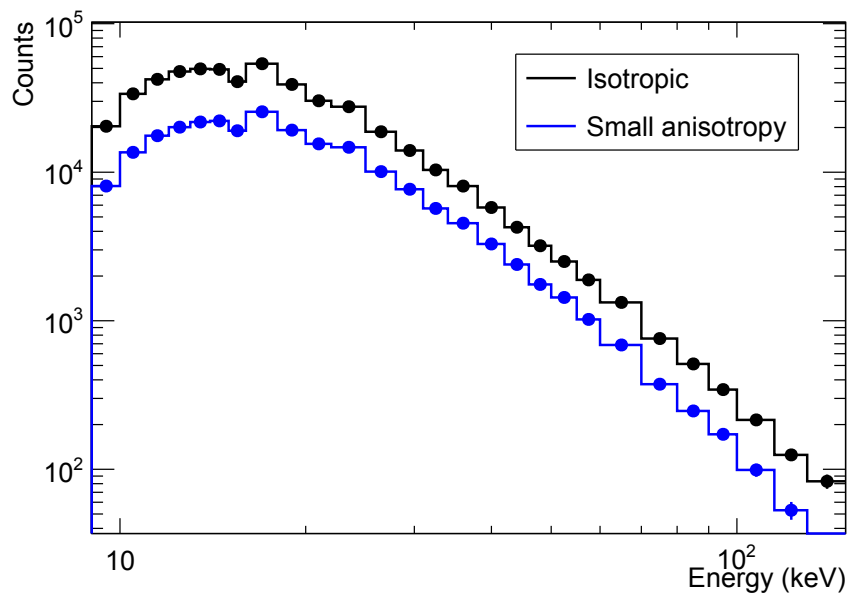

Fig. 9. Simulated measurements by STIX and MiSolFA within 1 min, with $\cos \theta_{\mathrm{S}}=0.7-0.8$ and $\cos \theta_{\mathrm{M}}=0.9-1.0$.

The second heuristic relationship connects the base-10 logarithm of the photon rate $R$ in $\mathrm{Hz} / \mathrm{cm}^{2}$ above $E_{\mathrm{thr}}$ to the flare intensity $I$. A parabolic fit with the function $R=q_{0}+q_{1} I+q_{2} I^{2}$ provides a very good description of this relationship, with bestfit parameters $q_{0}=3.107 \pm 0.009, q_{1}=1.154 \pm 0.010$, and $q_{2}=0.059 \pm 0.005$. The fit quality is very good, with $\chi^{2}=0.008$ over 12 degrees of freedom. Again, this is mostly a result of the large spread of flare characteristics.

The same relationships can also be exploited for MiSolFA, after accounting for the instrumental differences mentioned above. To put ourselves in the worst case, we considered the detection of a flare with STIX at perihelion (where it spends only a 


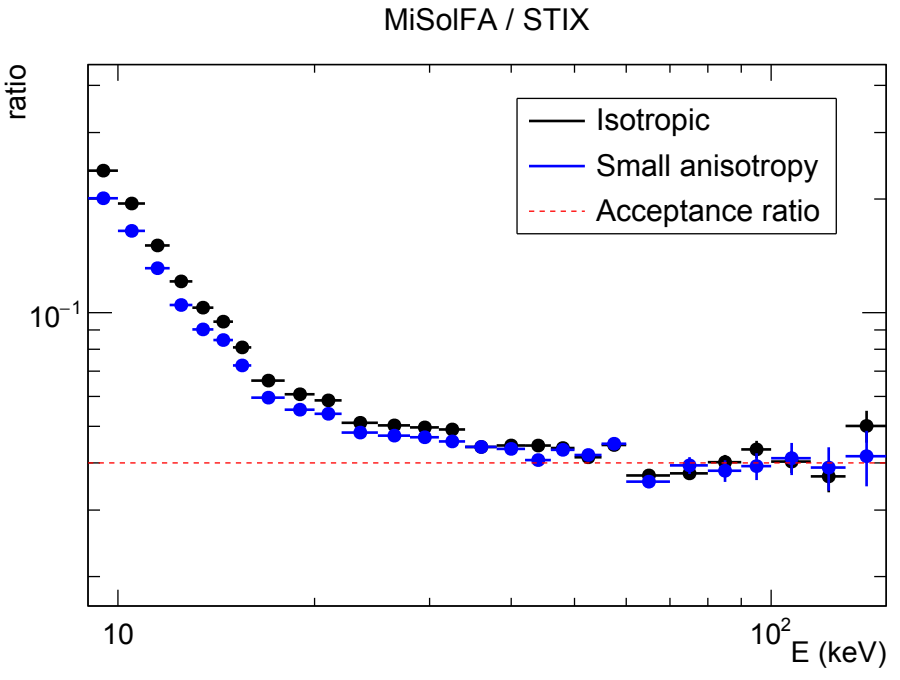

Fig. 10. Binwise ratio between the simulated measurements of MiSolFA and STIX, for fully isotropic and mildly anisotropic parent electron distributions, and a small angular separation $\left(\cos \theta_{\mathrm{S}}=0.7-0.8\right.$ and $\cos \theta_{\mathrm{M}}=0.9-1.0$ ).

Table 1. Expected non-thermal counts for a photon spectral index of $\gamma=4$ for MiSolFA.

\begin{tabular}{c|rrrr|rrrr}
\hline \hline$E$ bin & \multicolumn{9}{|c|}{ MiSolFA counts/min } & \multicolumn{4}{|c}{ Relative uncertainty } \\
$(\mathrm{keV})$ & M1 & M3 & M5 & X1 & M1 & M3 & M5 & X1 \\
\hline $28-31$ & 64 & & & & 0.13 & & & \\
$31-34$ & 43 & & & & 0.15 & & & \\
$34-38$ & 38 & 242 & & & 0.16 & 0.06 & & \\
$38-42$ & 25 & 159 & 402 & 1021 & 0.20 & 0.08 & 0.05 & 0.03 \\
$42-46$ & 17 & 108 & 274 & 696 & 0.24 & 0.10 & 0.06 & 0.04 \\
$46-50$ & 12 & 76 & 192 & 488 & 0.29 & 0.11 & 0.07 & 0.05 \\
$50-55$ & 11 & 68 & 171 & 435 & 0.31 & 0.12 & 0.08 & 0.05 \\
$55-60$ & 7 & 46 & 118 & 298 & 0.37 & 0.15 & 0.09 & 0.06 \\
$60-70$ & 9 & 57 & 145 & 368 & 0.33 & 0.13 & 0.08 & 0.05 \\
$70-80$ & 5 & 32 & 82 & 208 & 0.44 & 0.18 & 0.11 & 0.07 \\
$80-90$ & 3 & 20 & 50 & 128 & 0.56 & 0.22 & 0.14 & 0.09 \\
$90-100$ & 2 & 12 & 31 & 79 & 0.72 & 0.28 & 0.18 & 0.11 \\
$100-115$ & 2 & 11 & 29 & 73 & 0.74 & 0.30 & 0.19 & 0.12 \\
$115-130$ & 1 & 7 & 18 & 45 & 0.95 & 0.38 & 0.24 & 0.15 \\
\hline sums: & 240 & 840 & 1512 & 3840 & & & & \\
\hline
\end{tabular}

Notes. The relative uncertainty is statistical only.

very short fraction of its orbit). With respect to the orbit average, the closest distance gives a significant intensity magnification, bringing the ratio between STIX and MiSolFA acceptances to the very conservative factor of 25 , which is used below. Thus, the rate $\operatorname{logarithm} R$ for MiSolFA is taken to be 1.4 units smaller than STIX, and the expected non-thermal counts for a power law model can be computed for any given spectral index. Here we take as an example, a photon spectral index $\gamma=4$ for each instrument, which is steeper than the photon spectrum considered above $^{5}$, to get a conservative estimate of the photon counts. The result is shown in Table 1 for MiSolFA.

\footnotetext{
5 Because of the albedo contribution, the photon spectrum is not a single power law. By fitting with this function over different energy intervals, one gets a spectral index ranging from about 2.6 at lower energies to about 3.2 at higher energies.
}

For an M1-class flare, STIX should collect about 6000 nonthermal counts in one minute at the peak, while MiSolFA in the same time is expected to see 240 counts. For an M3-class flare, the expected counts per minute are 21000 for STIX and 840 for MiSolFA. For M5 and X1 classes, the expectation is 38k and $96 \mathrm{k}$ counts per minute for STIX, and $1.5 \mathrm{k}$ and $3.8 \mathrm{k}$ for MiSolFA. As STIX will collect many more events in each energy bin, the relative uncertainty in the ratio with MiSolFA is dominated by the counts of the latter instrument. This uncertainty (ignoring any systematic effect, which might be discovered in the future) is also reported in Table 1.

All M and X class flares will be suitable for directivity measurements. For the weaker M-class flares it might be beneficial to adopt a coarser energy binning, to decrease the statistical uncertainty in each bin. However, shape differences like those expected from the isotropic and mildly anisotropic models considered above can be measured without rebinning from class M3 above.

The last step is to estimate the expected number of flares of class M1 or higher, at the next solar maximum. The statistical distribution of solar flare classes is well described by a power law behaviour, with spectral index of about 2.1 and percent-level variations in slope across different solar cycles. According to the recent review by Winter \& Balasubramaniam (2015), one would expect to see at least 20 solar flares with class M1 or above per month during a solar maximum period. Together with our conservative estimate of the total overlapping live time of two months for STIX and MiSolFA, this implies that there should be at least 40 observations suitable for directivity measurement. Observing even one suitable flare, in which non-isotropic emission is detected and its dependence on energy is studied, would be a definite step forwards in our understanding of electron anisotropy in solar flares, and hence the expected number of good flares is very encouraging.

\section{Summary}

In this work, dual X-ray observations of the same solar flare from two upcoming instruments, STIX and MiSolFA, at different viewing angles are considered, in the context of prospective electron directivity measurements. A number of instrumental effects have been taken into account by performing a simulation of the response of STIX and MiSolFA to the photon fluxes computed in two models, in which the accelerated electrons are either fully isotropic or close to isotropic, helping us to determine the capabilities of the instrumentation for electron directivity measurements. The X-ray albedo component is also taken into account, as described in Jeffrey \& Kontar (2011).

Our study focussed on the energy range of the non-thermal component up to the energy bins in which the background counts are no longer negligible compared to the photon rate. Depending on the flare, this region goes from $20-30 \mathrm{keV}$ to about $100 \mathrm{keV}$. In order to have enough counts in MiSolFA, which is the instrument with the smaller acceptance, flares of class M1 or higher are required, to be able to distinguish between the two considered models. For such flares, we find that even for a mildly anisotropic case, and for spacecraft separations as small as 20$30^{\circ}$, STIX and MiSolFA will be able to detect shape differences in the X-ray spectra. Hence, higher levels of X-ray anisotropy should be easily detectable. Given the rate of flares as a function of the GOES class, and conservatively assuming a $8 \%$ net overlapping time for STIX and MiSolFA, one expects to observe at least 40 flares of M1 class or above during solar maximum, and 
it will be possible to perform quantitative estimates of the X-ray intensity along different directions, as a function of energy.

Therefore, the result of this study is that there will be at least 40 flares suitable for directivity studies, thanks to the stereoscopic measurements by STIX and MiSolFA at the next solar maximum. The combined use of these two instruments will allow for a quantitative measurement of solar flare electron anisotropy for the first time in solar flare physics, a vital diagnostic tool for understanding and constraining fundamental solar flare models of particle acceleration and transport.

Acknowledgements. The authors wish to thank Gordon Hurford for his help on the simulation of the flares distribution for STIX. N.L.S.J. and E.P.K. acknowledge support from STFC Consolidated Grant. The N.L.S.J. research leading to these results has received funding from the European Community's Seventh Framework Programme (FP7/2007-2013) under grant agreement No. 606862 (F-CHROMA).

\section{References}

Bai, T., \& Ramaty, R. 1978, ApJ, 219, 705

Battaglia, M., Grigis, P. C., \& Benz, A. O. 2005, A\&A, 439, 737

Benz, A. O. 2008, Liv. Rev. Sol. Phys., 5, 1

Brown, J. C. 1971, Sol. Phys., 18, 489

Brown, J. C., Emslie, A. G., \& Kontar, E. P. 2003, ApJ, 595, L115

Casadei, D. 2014, Proc. 2014 Nucl. Sci. Symp. and Med. Im. Conf., Seattle, USA

Chen, Q., \& Petrosian, V. 2013, ApJ, 777, 33

Dickson, E. C. M., \& Kontar, E. P. 2013, Sol. Phys., 284, 405

Eisen, Y., Evans, L., Floyd, S., et al. 2002, Nucl. Instrum. Methods A, 491, 176

Emslie, A. G., \& Brown, J. C. 1980, ApJ, 237, 1015

Emslie, A. G., Kontar, E. P., Krucker, S., \& Lin, R. P. 2003, ApJ, 595, L107

Emslie, A. G., Bradsher, H. L., \& McConnell, M. L. 2008, ApJ, 674, 570

Hajdas, W. E. A. 2015, J. Appl. Math. Phys., 3, 272

Harrison, F. A., Craig, W. W., Christensen, F. E., et al. 2013, ApJ, 770, 103

Holman, G. D., Aschwanden, M. J., Aurass, H., et al. 2011, Space Sci. Rev., 159, 107

Hurford, G. J., Schmahl, E. J., Schwartz, R. A., et al. 2002, Sol. Phys., 210, 61

Hurley, K. 1986, in The Sun and the Heliosphere in Three Dimensions, ed. R. G. Marsden, Astrophys. Space Sci. Lib., 123, 73
Jeffrey, N. L. S., \& Kontar, E. P. 2011, A\&A, 536, A93

Jeffrey, N. L. S., Kontar, E. P., Bian, N. H., \& Emslie, A. G. 2014, ApJ, 787, 86 Kane, S. R. 1981, Ap\&SS, 75, 163

Kane, S. R., Fenimore, E. E., Klebesadel, R. W., \& Laros, J. G. 1988, ApJ, 326, 1017

Kane, S. R., Hurley, K., McTiernan, J. M., et al. 1998, ApJ, 500, 1003

Kašparová, J., Karlický, M., Kontar, E. P., Schwartz, R. A., \& Dennis, B. R. 2005, Sol. Phys., 232, 63

Kašparová, J., Kontar, E. P., \& Brown, J. C. 2007, A\&A, 466, 705

Kontar, E. P., \& Brown, J. C. 2006, ApJ, 653, L149

Kontar, E. P., \& Jeffrey, N. L. S. 2010, A\&A, 513, L2

Kontar, E. P., Brown, J. C., Emslie, A. G., et al. 2011, Space Sci. Rev., 159, 301

Kontar, E. P., Bian, N. H., Emslie, A. G., \& Vilmer, N. 2014, ApJ, 780, 176

Krucker, S., \& Lin, R. P. 2008, ApJ, 673, 1181

Krucker, S., Benz, A. O., Hurford, G. J., et al. 2013, Nucl. Inst. Meth. Phys. Res. A, 732, 295

Leach, J., \& Petrosian, V. 1983, ApJ, 269, 715

Lin, R. P., Dennis, B. R., Hurford, G. J., et al. 2002, Sol. Phys., 210, 3

Marshall, F. E., Boldt, E. A., Holt, S. S., et al. 1980, ApJ, 235, 4

Massone, A. M., Emslie, A. G., Kontar, E. P., et al. 2004, ApJ, 613, 1233

McConnell, M. L., Smith, D. M., Emslie, A. G., et al. 2004, Adv. Space Res., 34, 462

McTiernan, J. M., \& Petrosian, V. 1990, ApJ, 359, 524

Melrose, D. B. 1994, ApJS, 90, 623

Meuris, A., Hurford, G., Bednarzik, M., et al. 2012, Nucl. Inst. Meth. Phys. Res. A, 695, 288

Miller, J. A., Larosa, T. N., \& Moore, R. L. 1996, ApJ, 461, 445

Müller, D., Marsden, R. G., St. Cyr, O. C., \& Gilbert, H. R. 2013, Sol. Phys., 285,25

Ohki, K.-I. 1969, Sol. Phys., 7, 260

Petrosian, V. 2012, Space Sci. Rev., 173, 535

Santangelo, N., Horstman, H., \& Horstman-Moretti, E. 1973, Sol. Phys., 29, 143

Simões, P. J. A., \& Kontar, E. P. 2013, A\&A, 551, A135

Suarez-Garcia, E., Hajdas, W., Wigger, C., et al. 2006, Sol. Phys., 239, 149

Tindo, I. P., Ivanov, V. D., Mandel'Stam, S. L., \& Shuryghin, A. I. 1970, Sol. Phys., 14, 204

Tomblin, F. F. 1972, ApJ, 171, 377

Winter, L. M., \& Balasubramaniam, K. 2015, Space Weather, 13, 286

Zanarini, M., Chirco, P., Dusi, W., et al. 2004, Nucl. Instrum. Methods A, 213, 315, 5th Topical Meeting on Industrial Radiation and Radioisotope Measurement Applications 